\title{
Are attentional dwell times inconsistent with serial visual search?
}

\author{
CATHLEEN M. MOORE and HOWARD EGETH \\ Johns Hopkins University, Baltimore, Maryland \\ and \\ LYNDON R. BERGLAN and STEVEN J. LUCK \\ University of Iowa, Iowa City, Iowa
}

\begin{abstract}
Duncan, Ward, and Shapiro (1994) estimated that attention must remain focused on an object for several hundred milliseconds before being shifted to another object, and they referred to this period as the attentional dwell time. An important implication of these long estimates of the dwell time for models of visual search is that the search process must not involve an item-by-item serial scanning mechanism. If it did, then searching through an array of items would require enormous amounts of time, whichbased on data from visual search experiments-it does not. The present report, however, provides evidence that the long estimates of attentional dwell time were caused, at least in part, by the use of masked targets. Implications of these variable estimates of the attentional dwell time for models of visual search are discussed.
\end{abstract}

Many theories of visual perception maintain that there are two distinct stages of visual information processing: an early unlimited-capacity stage and a later limitedcapacity stage (e.g., Duncan \& Humphreys, 1989; Hoffman, 1979; Neisser, 1967; Treisman \& Gelade, 1980; Wolfe, 1994). Although there is some agreement concerning the properties of the first stage, there is considerable disagreement concerning the nature of the second stage. Some theories maintain that, during the second stage, object identification is achieved by focusing attention on one item at a time (i.e., serial processing; see, e.g., Treisman \& Gelade, 1980; Treisman \& Sato, 1990; Wolfe, 1994). Other theories maintain that multiple items can be processed simultaneously during the second stage, but that for each item added to the display, less processing capacity is available for any given item (i.e., limited-capacity parallel processing; see, e.g., Bundesen, 1990; Duncan \& Humphreys, 1989).

Distinguishing empirically between serial and limitedcapacity parallel processing has proven difficult. The most common approach is to measure reaction time (RT) as a function of the number of items in the display (the display size) in visual search tasks. If search is serial and

This research was supported by NIH Grant 5 T32 MH18215 awarded to the Department of Psychology at Johns Hopkins University, Grant 95-38 from the McDonnell-Pew Program in Cognitive Neuroscience awarded to S.J.L, and NSF Grant SBR-9319356 awarded to H.E. Thanks are extended to John Duncan, Rich Schweickert, Jim Townsend, and Jeremy Wolfe for providing helpful comments on a previous draft. Correspondence should be addressed to $\mathrm{C}$. M. Moore, who is now at the Department of Psychology, Pennsylvania State University, University Park, PA 16802 or to S. J. Luck, Department of Psychology, University of lowa, 11 Seashore Hall E, lowa City, IA 52242-1407 (e-mail: steven-luck@uiowa.edu). self-terminating, then RT will increase as a linear function of display size and the slope of the RT $\times$ displaysize function will be twice as great on target-absent trials as on target-present trials (Sternberg, 1969; Treisman \& Gelade, 1980). This pattern of results has been observed frequently in visual search experiments and has been interpreted as evidence that attentive visual processing operates serially, dwelling on each item in the array for approximately $50 \mathrm{msec}$ (see Wolfe, 1994, for a review). Certain limited-capacity parallel models, however, predict exactly the same pattern of results (Townsend, 1972, 1990). Therefore, this pattern cannot be taken as strictly diagnostic of serial processing. Other methods have been developed to distinguish between serial and limitedcapacity parallel processing, but no consensus has been reached concerning the nature of second-stage processing (Egeth \& Dagenbach, 1991; Luck \& Hillyard, 1990; Townsend, 1990).

An alternative approach to this problem was recently reported by Duncan, Ward, and Shapiro (1994; Ward, Duncan, \& Shapiro, 1996; see also Eriksen \& Spencer, 1969; Krumhansl, 1977; Pashler \& Badgio, 1987; Shiffrin \& Gardner, 1972; Townsend \& Ashby, 1983). Using a dualtask paradigm, they estimated that attention must remain focused on an object for several hundred milliseconds before being shifted to another object. Given this long attentional dwell time, as this period was called, a serial search of even a moderate-size array (e.g., 12 items) would require several seconds of search time. Because search within such arrays can usually be completed in 600 $1,200 \mathrm{msec}$ (Wolfe, 1994), Duncan et al. argued that visual search could not possibly be conducted by a highspeed serial scanning mechanism.

Duncan et al. (1994; Ward et al., 1996) obtained their estimate of the attentional dwell time in a dual-task par- 
adigm in which two targets were presented in two different locations on each trial (see Figure 1A). Each target was followed by a mask, and subjects were required to identify both targets at the end of each trial. The two targets were usually presented successively, and the stimulus onset asynchrony (SOA) between them was varied between 0 and $1,000 \mathrm{msec}$. We will refer to the first target as "T1" and the second target as "T2." Duncan et al. found that identification accuracy was impaired for $\mathrm{T} 2$ at short SOAs but not at long SOAs. They interpreted this impairment in $\mathrm{T} 2$ accuracy as indicating that attention was still focused on $\mathrm{T} 1$ at short SOAs. According to this logic, then, the attentional dwell time for $\mathrm{T} 1$ could be estimated by determining the SOA at which T2 identification was no longer impaired. T2 accuracy was found to be impaired at SOAs of $450 \mathrm{msec}$ or less, implying that the attentional dwell time is approximately half a second.

In using this estimate of the attentional dwell time as evidence against high-speed serial models of visual search, Duncan et al. (1994; Ward et al., 1996) implicitly assumed that dwell time was constant and that the estimates obtained from their dual-task paradigm could be applied directly to the visual search paradigm. It is possible, however, that the attentional dwell time depends on the specific stimuli and task that are used. Thus, the attentional dwell time in typical visual search tasks might be substantially shorter than the half-second value implied by Duncan et al.'s data, and perhaps even fast enough to be consistent with typical high-speed serial search models.
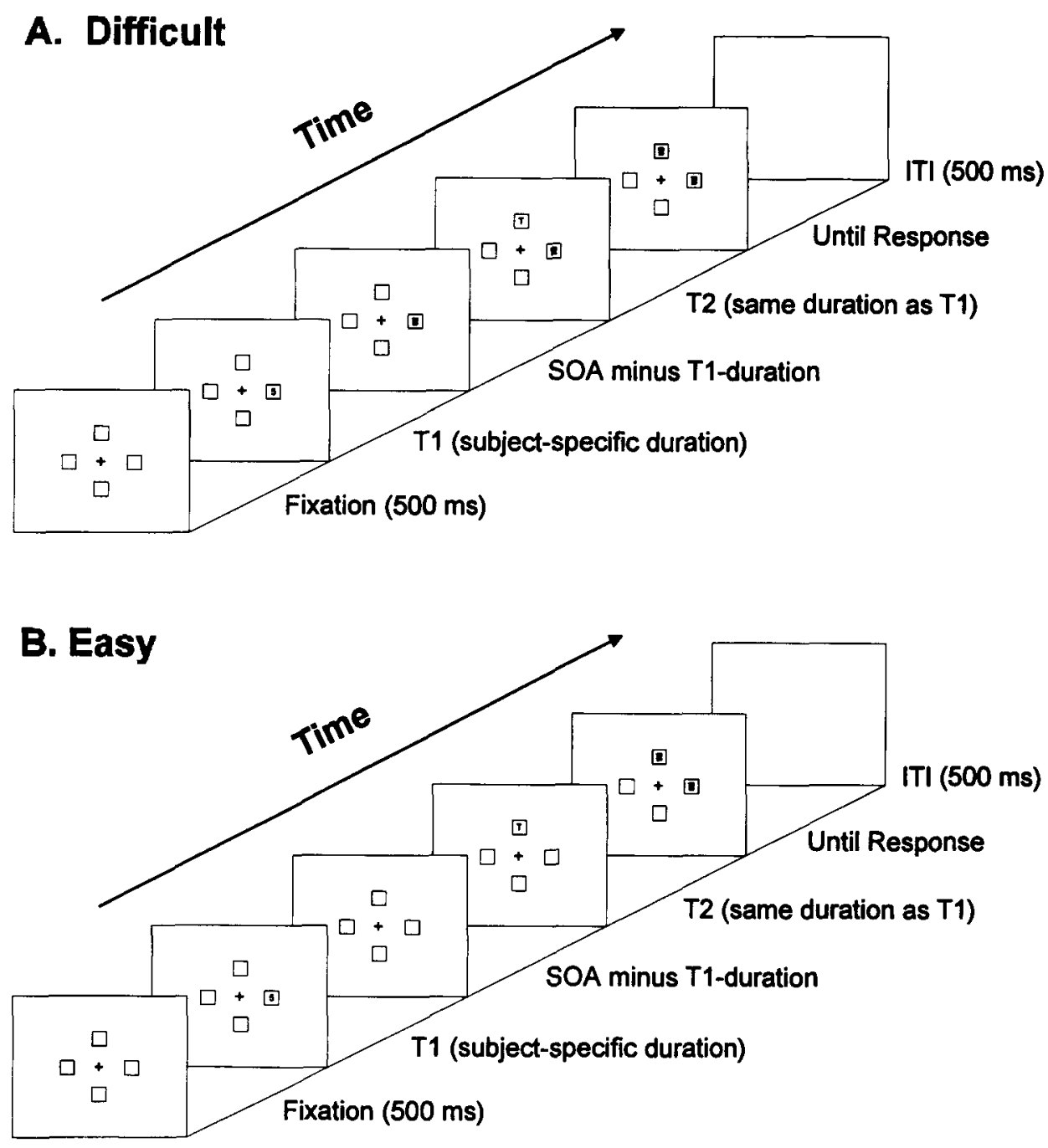

Figure 1. Typical sequence of stimuli on a single trial in Experiment 1. Each trial began with the presentation of a fixation point and the placeholders. After a 500-msec delay, the first target (T1) was presented for a subject-specific duration (see text). In the Difficult condition (Panel A), T1 was followed immediately by a mask, which remained present until the end of the trial. In the Easy condition (Panel B), the T1 mask was delayed until the onset of the T2 mask. T2 was presented with a variable SOA and was followed immediately by a mask which remained present until the end of the trial. Following the subject's responses, the screen became blank, and after $500 \mathrm{msec}$ the next trial began. 
There were several differences between the Duncan et al. (1994) paradigm and the typical visual search paradigm that could have inflated their estimate of the attentional dwell time. Although Duncan et al. showed that many of these differences do not influence the dwell time, there is one important attribute of their design that they did not examine, namely the use of masks following each target. It is reasonable to suppose that attention remains focused longer for difficult discriminations than for easy discriminations and that the use of masks may have made the discrimination very difficult, thus creating an unusually long dwell time. If this were true, the long dwell time that was estimated by Duncan et al. would not serve as definitive evidence against standard high-speed serial models of visual search, because it might not be an accurate estimate of the dwell time within visual search paradigms, in which stimuli are typically not masked. The purpose of the present study was to assess the effects of masking and thereby determine whether the attentional dwell time is constant, as implicitly assumed by Duncan et al., or whether the elimination of masking would lead to a shorter dwell time that would be more consistent with serial processing models.

Two experiments were conducted using slightly modified versions of the Duncan et al. (1994) dual-task paradigm. Both experiments manipulated the masking of the first target (the second target was always masked to avoid ceiling effects in T2 accuracy, which was the primary dependent variable). The two experiments were conducted independently, with somewhat different methods and in separate laboratories. This provides some assurance concerning the generality of the results, which is especially important because the implications of Duncan et al. represent an important challenge to widely held views of attention.

\section{METHOD}

\section{Subjects}

The subjects were 24 students at Johns Hopkins University (Experiment 1) and 20 students at the University of Iowa (Experiment 2); they were $18-31$ years of age.

\section{Stimuli}

Figure 1 illustrates the stimuli and procedure used in Experiment 1 (differences between experiments are noted below). In both experiments, a central fixation point and four placeholders-drawn at the 12 , 3,6 , and 90 'clock positions of an imaginary circle-were present throughout each trial. In Experiment 1, the placeholders were $1.4^{\circ} \times$ $1.4^{\circ}$ frames that were drawn in gray on a black background, and the entire display subtended $5.7^{\circ} \times 5.7^{\circ}$. In Experiment 2, the placeholders were $1.2^{\circ} \times 1.2^{\circ}$ frames that were drawn in black on a gray background, and the entire display subtended $8.0^{\circ} \times 8.0^{\circ}$. The targets were sans-serif uppercase letters ( $\mathrm{L}$ and $\mathrm{T}$ ) and digits ( 2 and 5 ); they subtended approximately $0.5^{\circ} \times 0.5^{\circ}$. The masks consisted of rectangular grids of randomly positioned white dots (Experiment 1 ) or of white line segments (Experiment 2), subtending approximately $0.7^{\circ} \times 0.7^{\circ}$.

\section{General Design}

In both experiments, two targets ( $\mathrm{T} 1$ and $\mathrm{T} 2$ ) were presented on each trial, and subjects were required to report the identity of both targets at the end of the trial (under no time pressure). The SOA between $\mathrm{T} 1$ and $\mathrm{T} 2$ varied randomly within trial blocks $(0,200,350,500,650,1,100$, and 1,300 msec in Experiment $\mathrm{l}$ and 0, 100, 200,300, 400, 500, 600, and 1,000 msec in Experiment 2). Both experiments included a "Difficult" condition in which both targets were followed immediately by a mask and an "Easy" condition in which the mask for the first target was either delayed (Experiment 1) or completely eliminated (Experiment 2). The Difficult and Easy conditions were mixed randomly within trial blocks in Experiment 1; they were run in separate, alternating trial blocks, and the order of conditions was counterbalanced across subjects in Experiment 2. Data were collected in 12 blocks of 56 trials each, providing 48 observations for each subject in each of the 14 combinations of SOA and masking condition in Experiment 1; they were collected in 6 blocks of 96 trials each, providing 36 observations for each subject in each of the 16 combinations of SOA and masking condition in Experiment 2.

\section{Procedure}

Experiment 1. Each trial in Experiment 1 began with a 500-msec presentation of the fixation point and placeholders. T1 was then presented for a subject-specific duration (mean $=60 \mathrm{msec}$ ), which was determined in an initial training period during which single targets $(2,5$, $\mathrm{L}$, or $\mathrm{T})$ were presented and target duration was adjusted until mean accuracy was in the range of $80 \%-90 \%$. In the Difficult condition, a mask was presented at the $\mathrm{T} 1$ location immediately at the offset of $\mathrm{Tl}$ and remained visible until the end of the trial. After a variable-duration SOA, T2 was presented for the same duration as T1. T2 was always followed immediately by a mask, which remained visible until the end of the trial. In the Easy condition, a mask was also presented at the location of $\mathrm{Tl}$ at the same time as the T2 mask, which was intended to discourage subjects from postponing identification of $\mathrm{T} 1$ until after $\mathrm{T} 2$ by using iconic memory. It should be noted that the Difficult and Easy conditions were identical when the SOA was $0 \mathrm{msec}$, and the data from these trials were therefore excluded from all analyses.

The position and order of the letter and digit stimuli were counterbalanced across subjects, such that $\mathrm{T} 1$ was a letter for some subjects and a digit for others and could appear at the 12 o'clock or 6 o'clock position for some subjects and at the $3 \mathrm{o}^{\prime}$ clock or 9 o'clock position for others. T2 was the opposite target type and was presented in one of the opposite pair of locations. The subjects made two 2 -alternative forced-choice responses to indicate the identity of $\mathrm{T} 1$ and $\mathrm{T} 2$. They were asked to wait until both $\mathrm{T} 1$ and $\mathrm{T} 2$ were presented before making any response and to make the $\mathrm{T} 1$ response before the $\mathrm{T} 2$ response. The screen became blank as soon as the subject responded and remained so for $500 \mathrm{msec}$, at which time the next trial began.

Experiment 2. The procedure for Experiment 2 was the same as that for Experiment 1 except for the following differences. Targets were always presented for a duration of $50 \mathrm{msec}$, and accuracy levels were maintained in the $70 \%-80 \%$ range by adjusting the brightness of the targets and masks (brightness levels of targets were equated across the Difficult and Easy conditions). Masks were presented for a duration of $100 \mathrm{msec}$, and no mask was ever presented in the T1 location in the Easy condition. T1 was always a letter and was presented at either the $30^{\circ}-$ clock or 9 o'clock position, selected at random on each trial; T2 was always a digit and was presented at either the $120^{\prime}$ 'clock or $60^{\prime}$ 'clock position, again selected at random. Finally, the placeholders remained visible at all times in this experiment, and a 4,000-msec response period separated the offset of the T2 mask and the beginning of the next trial, which was signaled by the onset of the fixation point.

\section{RESULTS}

Figure 2 summarizes the discrimination performance for both the first and second targets. In both experiments, the first target was discriminated substantially more accurately in the Easy condition than in the Difficult condition, indicating that the masking manipulations were successful in influencing the difficulty of the $\mathrm{T} 1 \mathrm{discrim}$ ination. There was little change in T1 accuracy across SOAs, however, which indicates that $\mathrm{T} 1$ was identified with very little interference from $\mathrm{T} 2$. 

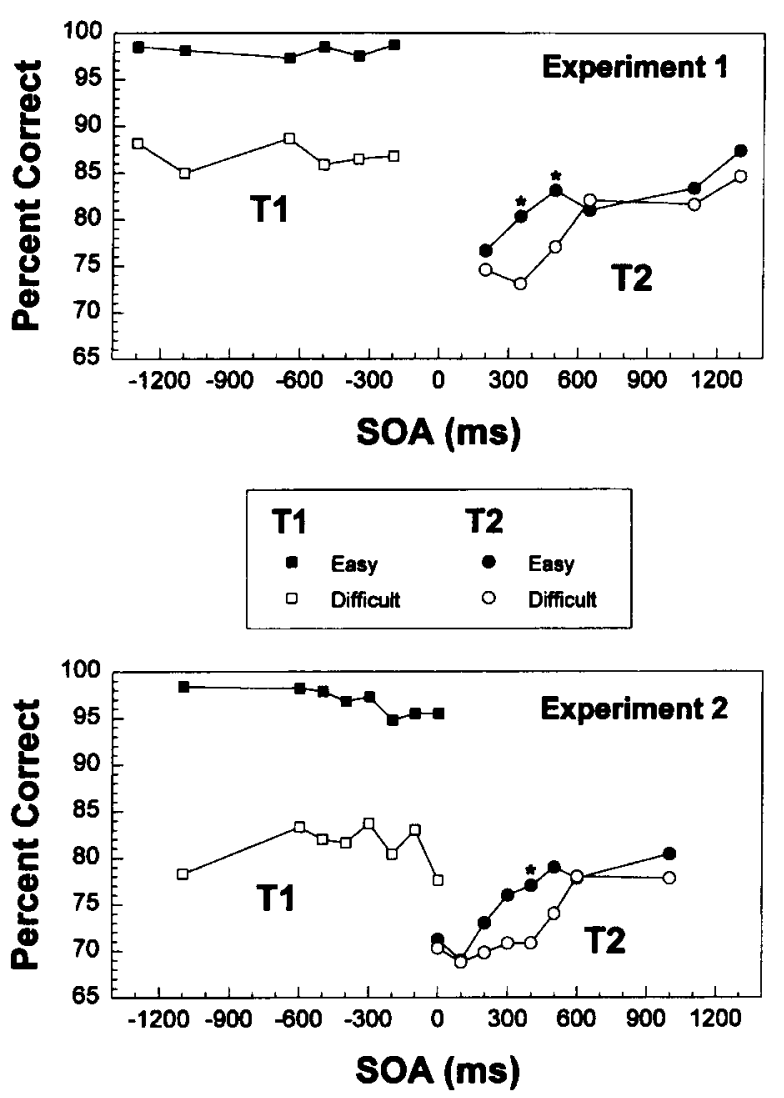

Figure 2. Percent correct as a function of SOA for the first target (T1) (squares) and T2 (circles) in Experiment 1 and Experiment 2. The filled symbols are the data from the Easy condition; the unfilled symbols are the data from the Difficult condition. Asterisks indicate significant differences between the Easy and Difficult conditions at the given SOA $(p<.01)$.

T2 discrimination, in contrast, was impaired at short SOAs, just as in Duncan et al.'s (1994) study. The time course of this impairment, however, was different in the Difficult and Easy conditions. Specifically, performance was impaired to the same level in both conditions at the shortest SOAs and recovered to the same asymptote level at the longest SOAs, but rose to asymptote sooner in the Easy condition than in the Difficult condition. Thus, the duration of the attentional dwell time appears to depend on whether or not the first target is masked.

\section{Analyses of Variance}

The accuracy data for $\mathrm{T} 1$ were submitted to separate two-way analyses of variance (ANOVAs) for the two experiments, with masking condition (Easy and Difficult) and SOA as within-subjects factors. In both experiments, the main effect of masking was significant [Experiment 1, $F(1,23)=39.48, p<.01$; Experiment $2, F(1,19)=185.80$, $p<.01]$. The main effect of SOA was not significant in Experiment $1[F(5,115)=1.87$, n.s.] but was in Experiment $2[F(7,133)=2.44, p<.05]$, apparently because of a slight increase in accuracy at longer SOAs. The masking $\times \mathrm{SOA}$ interaction was not significant for Experi- ment $2[F(7,133)=1.48$, n.s. $]$ but did reach significance in Experiment $1[F(5,115)=2.42, p<.05]$, although there was no easily interpretable pattern of interaction in the means. ${ }^{1}$ Planned comparisons confirmed that $\mathrm{T} 1$ identification was more accurate in the Easy condition than in the Difficult condition at all six of the nonzero SOAs in Experiment 1 and at all eight SOAs in Experiment 2 $(p<.01$ in every case).

Analogous ANOVAs were conducted for the T2 accuracy data. In both experiments, responses to the second target were generally more accurate following an Easy T1 than following a Difficult $\mathrm{T} 1$, which led to a significant main effect of masking the first target [Experiment 1, $F(1,23)=15.06, p<.01 ;$ Experiment $2, F(1,19)=4.52$, $p<.05]$. The main effect of SOA was also significant in both experiments [Experiment $1, F(5,115)=10.84, p<$ .01 ; Experiment $2, F(7,133)=6.92, p<.001$ ], reflecting a general trend toward greater accuracy at longer SOAs. The masking $\times$ SOA interaction did not reach significance in either experiment, although it was marginally significant in Experiment 1 [Experiment $1, F(5,115)=2.06$, $p<.08$; Experiment $2, F(7,133)=1.65$, n.s.].

Planned comparisons between the Easy and Difficult conditions were conducted for each SOA, although these must be interpreted with some caution due to the lack of a significant masking $\times$ SOA interaction. These comparisons indicated that performance began to improve sooner in the Easy condition than in the Difficult condition. Specifically, there were no significant differences in either experiment for SOAs less than $300 \mathrm{msec}$ or greater than $500 \mathrm{msec}(p>.15)$, but accuracy was significantly greater in the Easy condition than in the Difficult condition at the $350-\mathrm{msec}[t(23)=2.9, p<.01]$ and $500-\mathrm{msec}[t(23)=$ $3.7, p<.01]$ SOAs in Experiment 1 and at the $400-\mathrm{msec}$ SOA in Experiment $2[t(19)=2.95, p<.01]$. The difference was also marginally significant at the $300-\mathrm{msec}$ $[t(19)=1.89, p<.08]$ and $500-\mathrm{msec}[t(19)=1.68, p<$ .12] SOAs in Experiment 2. Combined with the similarity of the results across the two independent experiments, these planned comparisons provide evidence that the attentional dwell time was shorter in the Easy condition than in the Difficult condition.

\section{Curve Fitting}

Analysis of variance and related techniques are primarily sensitive to vertical displacements in the dependent variable, but the relevant issue in the present experiment is whether masking influenced the accuracy $\times$ SOA function in the horizontal dimension of time, with the upswing in accuracy occurring at an SOA that was shorter in the Easy condition than in the Difficult condition. To test this more specific question, we fit ogive-normal curves to each subject's accuracy $\times$ SOA function in the Difficult and Easy conditions and determined whether there was a horizontal shift in the point of inflection in these functions. The curve fitting was conducted with a simplex algorithm that minimized the mean-square error between the curve and the observed accuracy values. This curvefitting procedure allowed the horizontal position of the 
accuracy $\times$ SOA function to be summarized by a single value - the point of inflection of the ogive-normal function-and therefore allowed a more specific and therefore more powerful test of our hypothesis than was provided by the analyses of variance. ${ }^{2}$

The accuracy of the curve-fitting was reasonably good, with average root-mean-square errors of $0.35 \%$ and $0.49 \%$ in the Easy and Difficult conditions of Experiment 1 and $1.41 \%$ and $0.69 \%$ in the Easy and Difficult conditions of Experiment 2 . After the curve-fitting was completed, a point-of-inflection value was computed for each subject in each of the two masking conditions, and these values were submitted to a separate paired $t$ test for each experiment. A large and significant difference in the inflection point was observed for both Experiment $1[t(23)=3.22, p<.01]$ and Experiment $2[t(19)=2.15, p<.05]$, with mean inflection points of 112 and $625 \mathrm{msec}$ for the Easy and Difficult conditions of Experiment 1 and 220 and $524 \mathrm{msec}$ for the Easy and Difficult conditions of Experiment 2. This significant change in the time course of the accuracy $\times$ SOA function provide additional evidence for an effect of masking on the attentional dwell time.

\section{DISCUSSION}

Based on estimates from a dual-task paradigm, Duncan et al. (1994; Ward et al., 1996) argued that the dwell time of attention was too long to be compatible with the high-speed serial models of visual search that are considered in the current literature (e.g., Treisman \& Sato, 1990; Wolfe, 1994). If it takes nearly half a second to reallocate attention from one object to another, then it would take several seconds to process most visual displays by means of a serial (item-by-item) mechanism. Because even difficult search displays do not require that much time to process, visual processing must not involve a pure item-by-item scanning mechanism.

Duncan et al.'s (1994) argument depends on the assumption that their estimate of the attentional dwell time is comparable to the dwell times that occur during visual search. The present results, however, undermine that assumption. Specifically, this study showed that the long attentional dwell time that was obtained by Duncan et al. depended on masking, and that the dwell time was substantially shorter when the first target was not masked or the mask was delayed. Because stimuli are typically presented without masks during visual search, it follows that the attentional dwell time during visual search is probably shorter than the dwell time estimated by Duncan et al.

Although we found a shorter attentional dwell time in the Easy condition than in the Difficult condition, it might be argued that the dwell time in the Easy condition was still far longer than would be expected on the basis of visual search data. In both experiments, performance was substantially impaired in the Easy condition at SOAs of up to $200 \mathrm{msec}$, suggesting that the attentional dwell time is at least $200 \mathrm{msec}$ even with a very easy perceptual task. One might argue that this is still too long to accommodate typical visual search results, because a dwell time of $200 \mathrm{msec}$ might be expected to lead to a $200-\mathrm{msec}$ increment in RT for each additional item in the display, which is far larger than the increments that are usually observed.

We have two responses to this argument. First, the experiments reported here demonstrate that the attentional dwell time depends on the stimulation conditions, and it is therefore plausible that the dwell time varies across paradigms as well. For example, the use of simultaneously presented stimulus arrays in the visual search paradigm might yield faster shifts of attention because a sequence of shifts can be programmed. at the onset of the display, whereas a shift cannot be programmed until the second target has appeared in the present paradigm. Similarly, simultaneously presented arrays provide an opportunity for the visual system to perform substantial parallel preprocessing before attention is focused on a given item, which may also reduce the dwell time in visual search tasks. As a result of these-and perhaps otherfeatures of the visual search paradigm, it may be inappropriate to apply estimates of dwell time from the present dual-task paradigm to that of visual search.

Our second argument concerns the relationship between $\mathrm{RT} \times$ displaysize functions and the dwell time of attention in the visual search paradigm itself. It is typically assumed that the slope of the RT $\times$ displaysize function on target-absent trials reflects the time spent on each item, and this slope is generally in the range of $15-50 \mathrm{msec}$ per item. The slope of the RT $\times$ display-size function, however, almost certainly underestimates the amount of time spent on each item, because not all items in a display are processed during visual search, even on target-absent trials. Specifically, preattentive information about features can be used to guide attention toward the most likely target items, allowing target absence to be determined without an exhaustive search (Egeth, Virzi, \& Garbart, 1984; Treisman \& Sato, 1990; Wolfe, Cave, \& Franzel, 1989). As a result, the slope from an RT $\times$ display-size function may be based on an overestimate of the number of items processed, and may therefore lead to an underestimate of the amount of time spent on a single item. Indeed, when the stimuli are designed to minimize feature-based guidance, RT $\times$ display-size slopes can reach $100 \mathrm{msec}$ per item (e.g., Bilsky \& Wolfe, 1995). Finally, even in the absence of preattentive guidance, subjects may frequently make a target-absent response before exhausting the entire array, especially when the array has many items. This assertion is supported by the almost universal findings of greater error rates at larger display sizes and larger numbers of misses than false alarms. ${ }^{3}$ Thus, the commonly cited slope of $50 \mathrm{msec}$ per item may substantially underestimate the actual dwell time.

What, then, is the dwell time of attention in visual search? The most direct interpretation of the present data would suggest a value of approximately $200 \mathrm{msec}$, at least for the usual case of unmasked stimuli. As discussed above, however, attention may operate more efficiently within visual search paradigms than within the sequential presentation paradigm used here. Consequently, the 200-msec estimate derived from the present data may be an overestimate. Likewise, the $50-\mathrm{msec}$ value that is commonly estimated from RT $\times$ display-size functions is probably an underestimate. We therefore suggest that the true dwell time probably lies somewhere between 50 and $200 \mathrm{msec}$. Standard serial models, such as those of Treisman (e.g., Treisman \& Sato, 1990) and Wolfe (e.g., 1994), could be adjusted to accommodate a dwell time in the 50- to 100-msec range, but could not easily accommodate a dwell time approaching $200 \mathrm{msec}$. It will therefore be important for future research to provide a more precise estimate of the actual dwell time in visual search

\section{REFERENCES}

Bilsky, A. B., \& WolfE, J. M. (1995). Part-whole information is useful in visual search for size $\times$ size but not orientation $X$ orientation conjunctions. Perception \& Psychophysics, 57, 749-760.

Bundesen, C. (1990). A theory of visual attention. Psychological Review, 97, 523-547.

DunCan, J., \& Humphreys, G. W. (1989). Visual search and stimulus similarity. Psychological Review, 96, 433-458.

Duncan, J., Ward, R., \& Shapiro, K. L. (1994). Direct measurement of attentional dwell time in human vision. Nature, 369, 313-315.

Egeth, H., \& DagenbaCh, D. (1991). Parallel versus serial processing in visual search: Further evidence from subadditive effects of visual quality. Journal of Experimental Psychology: Human Perception \& Performance, 17, 551-560.

EgEth, H. E., Virzi, R. A., \& Garbart, H. (1984). Searching for conjunctively defined targets. Journal of Experimental Psychology: Human Perception \& Performance, 10, 32-39.

ERIKSEN, C. W., \& SPENCER, T. (1969). Rate of information processing in visual perception: Some results and methodological considerations. Journal of Experimental Psychology Monographs, 79 (2, Pt. 2), $1-16$.

Hoffman, J. E. (1979). A two-stage model of visual search. Perception \& Psychophysics, 23, 1-11.

KRUMHANSL, C. L. (1977). Naming and locating simultaneously and sequentially presented letters. Perception \& Psychophysics, 22, 293-302. 
LuCK, S. J., \& Hillyard, S. A. (1990). Electrophysiological evidence for parallel and serial processing during visual search. Perception \& Psychophysics, 48, 603-617.

NeISSER, U. (1967). Cognitive psychology. New York: Appleton-CenturyCrofts.

Palmer, J., Ames, C. T., \& Lindsey, D. T. (1993). Measuring the effect of attention on simple visual search. Journal of Experimental Psychology: Human Perception \& Performance, 19, 108-130.

Pashler, H., \& BaDglo, P. C. (1987). Attentional issues in the identification of alphanumeric characters. In M. Coltheart (Ed.), Attention and performance XII (pp. 63-81). Hillsdale, NJ: Erlbaum.

ShIFFrin, R. M., \& GARDNER, G. T. (1972). Visual processing capacity and attentional control. Journal of Experimental Psychology, 93, 72-83.

STERNBERG, S. (1969). Memory-scanning: Mental processes revealed by reaction-time experiments. American Scientist, 57, 421-457.

TowNSEND, J. T. (1972). Some results concerning the identifiability of parallel and serial processes. British Journal of Statistical Psychology, 25, 168-199.

TOWNSEND, J. T. (1990). Serial vs. parallel processing: Sometimes they look like Tweedledum and Tweedledee, but they can (and should) be distinguished. Psychological Science, 1, 46-54.

TOWNSEND, J. T., \& ASHBY, F. G. (1983). Stochastic modelling of elementary psychological processes. Cambridge: Cambridge University Press.

Treisman, A. M., \& Gelade, G. (1980). A feature-integration theory of attention. Cognitive Psychology, 12, 97-136.

Treisman, A. M., \& Sato, S. (1990). Conjunction search revisited. Journal of Experimental Psychology: Human Perception \& Performance, 16, 459-478.

WARD, R., DunCaN, J., \& ShaPIRo, K. (1996). The slow time-course of visual attention. Cognitive Psychology, 30, 79-109.
WoLFE, J, M. (1994). Guided Search 2.0: A revised model of visual search. Psychonomic Bulletin \& Review, 1, 202-238.

Wolfe, J. M., CAVE, K. R., \& Franzel, S. L. (1989). Guided search: An alternative to the feature integration model for visual search. Journal of Experimental Psychology: Human Perception \& Performance, 15, 419-493.

\section{NOTES}

1. Visual inspection of the figure suggests that the interaction may have arisen from a smaller masking effect at the $650-\mathrm{msec}$ SOA $(-650 \mathrm{msec}$ in Figure 2a, Experiment 1). We have no explanation for why the effect of masking should have been smaller at this SOA than at the other SOAs.

2. This analysis is not meant to imply that the shape of the accuracy $\times$ SOA curves was precisely ogive normal or that the influence of masking consisted of a pure shift in the horizontal dimension. Rather, ogive-normal functions were used as a convenient method of providing a single parameter (the inflection point) that could be used to test the specific hypothesis of a change in the time course of the accuracy $x$ SOA function. A specific test of this nature is typically more powerful than the more general interaction test in an ANOVA, which is capable of detecting many possible patterns of interaction but is typically less sensitive to any particular pattern than is a specific test of that pattern.

3. It should be noted that other factors may also contribute to these error effects (see Palmer, Ames, \& Lindsey, 1993).

(Manuscript received October 19, 1995; revision accepted for publication March 30, 1996.) 